AUTORES:

Naerton J. Xavier Isidoro

Maria L. F. L. Cavalcante

Victor E. da Silva Coimbra

Cícera K. Furtado Ramos

Universidade Regional do Cariri, Brasil

p-194-203, 2000

2. CARVALHO, Y. O "mito" da atividade física e saúde. 3

ed. São Paulo: Hucitec, 2001.

3. CASPERSEN, C. J.; POWELL, K. E.; CHRISTENSON,

G. M. Physical activity, exercise, and Physical fitness:

definitions and distinction for health-related research.

Public Health Reports, V.100, n.2 p-126-131, 1985

4. GROPPA, J. (Org.). Indisciplina na Escola: Alterna-

tivas Teóricas e Práticas, São Paulo: Summus, 1996

5. GUEDES, E. Curso de metodologia científica. HD li-

vros editora, Curitiba, 1997

6. JOVCHELOVITCH, S. Os contextos do saber: representações, comunidade e cultura. Petrópolis. RJ:

Vozes, 2008

7. MARTINS, R. Exercício Físico e Saúde Pública. Lisboa: Livros Horizonte, 2006

8. MOSCOVICI, S. Representações sociais: investigações em psicologia social. 6a ed. Vozes, Rio de Janeiro, 2009.

9. SETTON, M. A teoria do habitus em Pierre Bourdieu:

uma leitura contemporânea. Revista Brasileira de

Educação, n. 20. maio/jun./jul./ago. de 2002.

RESUMO
Avaliação da aptidão física

funcional de idosos praticantes

de atividade física na cidade

\section{do Crato-CE}

PALAVRAS CHAVE:

Idoso. Aptidão física. Exercício físico.

0 presente estudo tem como objetivo principal avaliar a aptidão funcional de idosos praticantes de atividade física em dois locais da cidade do Crato-CE: NASF 2 e Associação Arte e Vida. Trata-se de um estudo descritivo, transversal e quantitativo. Para esta investigação científica foram selecionados 33 idosos, sendo 31 do sexo feminino e 2 do sexo masculino praticantes de atividade física. Aplicou-se um questionário estruturado pelos autores em que foram pesquisados os seguintes itens: idade, sexo e medidas antropométricas. Posteriormente, foi aplicada uma bateria de testes para avaliar a aptidão física de idosos mensurando-se: força dos membros inferiores, força dos membros superiores, resistência aeróbia, flexibilidade dos membros superiores, flexibilidade dos membros inferiores, agilidade e equilíbrio. Concluiu-se que a prática regular de exercícios físicos para a maioria dos idosos pesquisados influenciou positivamente no tocante à força de membros superiores e inferiores. Embora uma considerável parcela da amostra tenha obtido valores satisfatórios nos demais itens analisados, observou-se, também, a necessidade de se reavaliar as atividades relacionadas à flexibilidade, resistência aeróbica, equilíbrio e agilidade. 
Assessment of the functional physical

fitness of elderly physical activity

practitioners in the city of Crato-CE

\section{ABSTRACT}

The present study aims: To assess the functional fitness of elderly practitioners of physical activity in two locations in the city of Crato - CE: NASF 2 and Association Art and Life. This is a descriptive, cross-sectional quantitative study. For this scientific research 33 seniors were selected, 31 were female and Male practitioners of physical activity 2. Applied a structured questionnaire by the authors in which the following items were investigated: age, sex, anthropometric measures. Subsequently, a battery of tests was administered to assess the physical fitness of elderly, measuring up: Lower Limb Strength, strength of upper limbs, aerobic endurance, flexibility, upper limb, lower limb flexibility, agility and balance. It was concluded that regular physical exercises for most seniors surveyed had a positive effect with regard to the strength of upper and lower limbs. Although a considerable portion of the sample has obtained satisfactory values in the other items analyzed, also observed the need to reassess the activities related to flexibility, aerobic, balance, endurance and agility.

\section{KEY WORDS}

Elderly. Fitness. Exercise.

\section{INTRODUÇÃo}

A sociedade moderna vem vivenciando nas últimas décadas um processo de envelhecimento populacional sem precedentes na história da humanidade. Este fenômeno mundial desencadeou um aumento na incidência de doenças crônico-degenerativas, comumente desenvolvidas em idades avançadas.

Essas enfermidades têm uma relação direta com o estilo de vida adotado pelo indivíduo e podem ter seus efeitos minimizados com a adoção de comportamentos positivos ao longo da vida que privilegiem a prática regular de atividades físicas, controle do estresse, uma dieta nutricional saudável e hábitos preventivos.

Especificamente, a prática regular de atividades físicas a partir do desenvolvimento de programas de exercícios físicos adaptados às demandas e possibilidades do idoso, contribui para um envelhecimento com mais qualidade de vida e saúde.

A adoção de um programa de exercícios físicos na terceira idade pode interferir positivamente na aptidão física do idoso, reduzindo a fragilidade física associada ao envelhecimento.

A elaboração desse programa de exercícios físicos depende de uma prévia avaliação física que seja capaz de delinear os pontos positivos e negativos em relação à condição física do geronte.

0 presente estudo tem como objetivo principal: Avaliar a aptidão funcional de idosos praticantes de atividade física em dois locais da cidade do Crato-CE: NASF 2 (Bairro São Miguel) e Associação Arte e Vida (Bairro Muriti)

A coleta de dados que fundamenta esta investigação científica envolveu Acadêmicos pertencentes ao Núcleo de Pesquisa, Estudo e Extensão em Educação Física - NUPEF sob a supervisão de um Professor-Orientador pertencente ao referido grupo científico.

\section{ENVELHECIMENTO}

E ATIVIDADE FÍSICA

Segundo estimativas do IBGE até 2025 a população brasileira terá em torno de $33 \mathrm{mi}$ Lhões de idosos. Um número superior a $13 \%$ da população estará, portanto, na faixa etária acima dos 60 anos.

Campos ${ }^{(2)}$ apresenta a seguinte classificação e nomenclaturas relacionadas ao envelhecimento proposta pela Organização Mundial da Saúde (OMS):

Indivíduos entre 45 - 59 anos são chamados "Meia Idade"

Indivíduos entre 60 - 74 anos são "Idosos"

Indivíduos entre 60-74 anos são "Velhos"

Indivíduos acima de 90 anos são chamados "Muito velhos"

Nahas ${ }^{(4)}$ ressalta que aspectos como a perda da independência, isolamento social em 
função da falta de apoio dos amigos e familiares assim como as restrições de ordem financeira são fatores que incidem negativamente na qualidade de vida do idoso.

0 referido autor, acrescenta que o problema de ordem econômica pode ser resolvido através de políticas públicas direcionadas aos gerontes, fruto da mobilização dos milhões de aposentados do nosso país.

Já questões que afetam a independência e autonomia dos idosos e a disposição para realizar as tarefas diárias podem ser influenciadas positivamente com a adoção de um estilo de vida ativo, baseado em comportamentos positivos relacionados à prática regular de exercícios físicos, alimentação adequada, controle do estresse e atitudes preventivas.

Rikli e Jones ${ }^{(5, p .3)}$ afirmam que "[...] Enquanto a tecnologia médica contribui para aumentar a expectativa de vida, a tecnologia de automação ou de informática leva a estilos de vida cada vez mais sedentários e ao aumento do risco de problemas crônicos de saúde e de mobilidade"

Especificamente, entre os fatores supracitados, a prática regular de exercícios físicos na terceira idade, compreendida como "[...] uma das formas de atividade física planejada, estruturada, repetitiva, que objetiva o desenvolvimento (ou manutenção) da aptidão física de habilidades motoras ou a reabilitação orgânico-funcional" (4, p.46), pode beneficiar esta população.

Para Dantas e Vale ${ }^{(3)}$, autonomia é classificada em três aspectos: autonomia de ação que se refere à noção de independência física, autonomia de vontade relacionada à possibilidade de autodeterminação e, por fim, autonomia de pensamentos que permite o indivíduo julgar qualquer situação.

As alterações funcionais ocorridas com os idosos ao passar dos anos e associadas às doenças crônicas não- transmissíveis têm tornado os idosos menos ativos e mais dependentes na realização das atividades da vida diária, comprometendo a sua qualidade de vida ${ }^{(1)}$.

TESTE DE APTIDÃO

FÍSICA PARA IDOSOS

0 teste de Aptidão Física para idosos desenvolvido por Rikli e Jones ${ }^{(5)}$ avalia a aptidão física funcional do idoso, observando os níveis adequados de força, resistência, agilidade, flexibilidade, capacidades estas essenciais para realizar as atividades da vida diária na terceira idade.

Conceitua-se Aptidão Física Funcional como "[...] capacidade física de realizar as atividades normais da vida diária de forma segura e independente, sem fadiga injustificada" (5).

Os testes propostos por Rikli e Jones ${ }^{(5)}$ são os seguintes:
TESTE DE LEVANTAR DA CADEIRA

B.JETIVO: Avaliar a força dos membros inferiores

PROCEDIMENTO: 0 participante é instruído a sentar no meio do assento com as costas eretas, pés fixos no chão e braços cruzados na altura dos punhos contra o tórax. Ao comando de "iniciar" o participante se levanta de forma completa e retorna à posição sentada. 0 idoso deve ser estimulado a se levantar e sentar o máximo de vezes possível em 30 segundos. Se o indivíduo tiver completado mais da metade do movimento de levantar da cadeira no final dos 30 segundos, conta-se como um movimento completo. 0 teste deve ser aplicado apenas uma vez.

TESTE DE FLEXÃO DE BRAÇO

OBJETIVO: Medir a Força dos Membros Superiores

PROCEDIMENTO: Pedir ao idoso para sentar-se em uma cadeira com espaldar reto, os pés fixos no chão e o lado dominante do corpo próximo à borda lateral do assento da cadeira. 0 braço cuja mão segura o peso deve ficar estendido ao longo do tronco, perpendicular ao chão. 0 participante deve segurar o peso como se estivesse apertando a mão de uma pessoa para cumprimentá-la. Partindo dessa posição, o braço é flexionado em direção ao ombro, com a palma da mão gradativamente se voltando para cima durante a flexão. 0 peso, então, retorna à posição completamente estendida com a mão na mesma posição de início. Ao comando "iniciar" o participante realiza a flexão de braço em amplitude total de movimento (de extensão completa à flexão completa) com o máximo de repetições em 30 segundos.

TESTE DE MARCHA ESTACIONÁRIA

OBJETIVO: Avaliar a resistência aeróbia

PROCEDIMENTO: Situa-se o geronte próximo a uma parede, uma porta ou a uma cadeira de encosto alto. Marca-se a altura mínima de elevação do joelho, que é um ponto central entre a patela e crista ilíaca. Ao comando de "iniciar" o participante começa a marchar no lugar (sem correr) tantas vezes quantas conseguir em um período de 2 minutos. Embora os dois joelhos devam se elevar à altura correta, deve-se usar o contador para registrar apenas 0 número de vezes em que o joelho direito atingiu a marca.

TESTE DE SENTAR E ALCANÇAR OS PÉS

OBJETIVO: avaliar a flexibilidade dos membros inferiores

PROCEDIMENTO: o participante deve sentar-se na beirada da cadeira. A dobra entre a superfície superior da coxa e a nádega deve coincidir com a beirada do assento da cadeira. Uma das pernas fica fletida com o pé fixo no chão, a outra perna é estendida o máximo possível na frente do quadril. 0 tornozelo é apoiado no chão, com o pé fletido a aproximadamente 90 graus. Com os braços estendidos para fora, as mãos sobrepostas e os dedos médios na mesma altura, o participante se curva lentamente para frente, flexionando a articulação do 
quadril para tentar alcançar ou ultrapassar os dedos pé. Se o joelho estendido começar a fletir, pedir ao participante para recuar as costas até que o joelho fique estendido. 0 alcance máximo deve ser sustentado durante 2 segundos. 0 participante deve praticar o teste em ambas as pernas para ver qual o melhor resultado. Medir a distância da ponta dos dedos até a ponta do tênis e anotar o número de centímetros mais próximo. 0 ponto central do tênis representa o ponto zero. Se as mãos não alcançarem esse ponto, registrar a distância como escore negativo (-); se os dedos médios tocarem os dedos do pé, registrar um escore de zero; e se passarem do ponto central registrar a distância como escore positivo (+).

TESTE DE ALCANÇAR AS COSTAS

OBJETIVO: Avaliar a flexibilidade dos membros superiores

PROCEDIMENTO: o participante deve ficar em pé e passar a mão selecionada sobre o mesmo ombro, com a palma da mão e os dedos estendidos, tentando alcançar a parte central das costas. Observar que o cotovelo deve apontar para cima. Pedir ao participante para colocar a outra mão em torno da parte posterior da cintura e com a palma da mão voltada para cima, tentando fazer com que os dedos de ambas as mãos se toquem ou sobreponham. São realizadas duas tentativas antes de registrar o escore. Aplicar o teste duas vezes. Anotar ambos os escores, levando-se em consideração o número de centímetros mais próximo. Medir a distância de sobreposição ou a distância entre as pontas dos dedos médios, indicando o melhor escore. Atribuir escore negativo (- ) se os dedos médios não se tocarem, escore zero se os dedos médios se tocarem ligeiramente e escore $(+)$ se os dedos médios se sobrepuserem.

\section{LEVANTAR E CAMINHAR}

OBJETIVO: avaliar a agilidade e o equilíbrio dinâmico

PROCEDIMENTO: instruir o participante para sentar na parte central do assento da cadeira com as costas eretas, os pés sobre o chão e as mãos sobre as coxas. Um pé deve se colocado ligeiramente à frente do outro, com o tronco levemente inclinado para frente. Ao comando "iniciar" o participante deve se levantar da cadeira, caminhar o mais rápido possível até o cone, contorná-lo e voltar para a cadeira, sentando-se novamente. Acionar o cronômetro ao sinal "iniciar", quer o participante começa a se movimentar ou não, e pará-lo no exato instante em que a pessoa sentar na cadeira outra vez. Após a demonstração da forma adequada e do ritmo desejado de teste, o participante deve praticar uma vez e depois fazer duas tentativas. Os dois tempos devem ser registrados, assinalando o melhor.

ALTURA E PESO (IMC)

OBJETIVO: Avaliar o índice de massa corporal (IMC)
PROCEDIMENTO: Em relação à medição da altura, o participante deve ficar com a parte posterior da cabeça contra a parede (a parte central da cabeça deve ficar alinhada com a fita métrica) e os olhos voltados diretamente para frente. Colocar a régua ( ou objeto semelhante) no topo da cabeça do participante e, sustentando-a no nível, encostá-la na fita métrica. A altura da pessoa correponde ao escore em centímetros. Quanto ao Peso, o participante deve remover peças de roupa que possam atrapalhar. Arredondar a medida de peso para o número mais próximo, descontando o peso do calçado. Registrar a altura e o peso do participante na ficha de escore. Para calcular o IMC, divide-se o peso em quilogramas pelo quadrado da altura IMC $=\mathrm{kg} / \mathrm{m}$

\section{METODOLOGIA}

TIPO DE ESTUDO

Este é um estudo descritivo, transversal e quantitativo, realizado com Idosos pertencentes ao Núcleo de Apoio à Saúde da Família (NASF) localizado no Bairro São Miguel e, também, com gerontes frequentadores da Associação Arte e vida, situada no Bairro Muriti, ambos localizados na Cidade do Crato-CE.

AMOSTRA

Para esta investigação científica foram selecionados 33 idosos, sendo 31 do Sexo feminino e 2 do Sexo Masculino, praticantes de atividade física em dois locais da cidade do CratoCE: Associação Arte e Vida e NASF 2.

TABELA 1 - Caracterização dos Participantes (n=33)

\begin{tabular}{ccc}
\hline & MÉDIA & DESVIO PADRÃO \\
\hline IDADE (ANOS) & 66,84 & 6,02 \\
\hline PESO (KG) & 65,06 & 10,33 \\
\hline ESTATURA (CM) & 1,53 & 0,05 \\
\hline IMC (KG/M) & 27,75 & 4,44 \\
\hline
\end{tabular}

CENÁRIO DA PESQUISA

Os Núcleos de Apoio à Saúde da Família (NASFs) foram criados pelo Ministério da Saúde 
em 2008 com o objetivo de apoiar a consolidação da Atenção Básica no Brasil, ampliando as ofertas de Saúde na rede de serviços. A cidade do Crato conta com NASFs que atuam nos Bairros Muriti, Seminário, Batateiras e São Miguel, sendo este último um dos locais da pesquisa em questão. O NASF 2 localizado Bairro São Miguel funciona com idosos do Centro de Saúde Teodorico Teles.

O outro local escolhido para a coleta dos dados foi a Associação Arte e Vida, organização comunitária criada no ano de 1999 por um grupo de três amigas residentes do Bairro Muriti da Cidade de Crato-CE com intuito de ajudar as pessoas carentes do seu bairro, em especial os idosos.

CRITÉRIOS DE INCLUSÃO

Foram selecionados 33 participantes de ambos os sexos pertencentes a dois projetos de atividade física desenvolvidos na cidade do Crato-CE, possuindo uma frequência às aulas mínima de duas vezes por semana.

\section{CUIDADOS ÉTICOS}

Os participantes contemplados na pesquisa assinaram um Termo de Consentimento Livre e Esclarecido (TCLE) , o qual trata das informações básicas sobre os testes aplicados, ressaltando os riscos, benefícios e sua aplicabilidade, assim como a finalidade da investigação científica no que concerne à sua utilização em eventos e publicações científicas.

PROCEDIMENTO DE COLETA DE DADOS

Inicialmente, aplicou-se um questionário estruturado pelos autores em que foram pesquisados os seguintes itens: idade, sexo, medidas antropométricas (peso, estatura, imc) Posteriormente, foi aplicada uma bateria de testes para avaliar a aptidão física de idosos, segundo o protocolo de Rikli e Jones ${ }^{(5)}$, mensurando-se: Força dos membros inferiores (teste de levantar da cadeira), força dos membros superiores (teste de flexão de braço) resistência aeróbia (teste de marcha estacionária), flexibilidade dos membros superiores (teste de alcançar as costas), flexibilidade dos membros inferiores ( teste de sentar e alcançar os pés), agilidade e equilíbrio (teste de levantar e caminhar). Os dados coletados foram tabulados e organizados no Excel 2010.

\section{RESULTADOS E DISCUSSÃO}

\section{DOS DADOS}

Os resultados do presente estudo foram baseados na Faixa Normal de Escores proposta por Rikli e Jones ${ }^{(5)}$ os quais, admitem como na "média" aqueles indivíduos que se encontravam entre estes valores. Escores inferiores a essa faixa em questão foram considerados como "abaixo da média" e aqueles acima dos valores descritos, "acima da média",

A Tabela 2 demonstra que a maioria dos idosos pesquisados encontra-se em níveis considerados normais ou mesmo acima da média no tocante à capacidades como força dos membros inferiores e superiores.

Nos locais pesquisados acontecem sessões com exercícios de musculação para os idosos. Os resultados favoráveis detectados no estudo em questão confirmam os benefícios advindos da prática de exercícios resistidos entre os gerontes.

Campos ${ }^{(2)}$ cita entre os ganhos advindos da prática da ginástica com pesos na terceira idade os seguintes aspectos: aumento da força muscular, aumento da potência muscular pequeno aumento da área de secção transversal, diminuição dos níveis de dor, aumento da capacidade funcional, melhoria da postura geral, aumento da agilidade, aumento da flexibilidade e aumento da resistência.

TABELA 2 - Faixas dos Escores Obtidos na Pesquisa ( $\mathrm{n}=33)$

\begin{tabular}{cccc}
\hline & ABAIXO DA MÉDIA & MÉDIA & ACIMA DA MÉDIA \\
\hline $\begin{array}{c}\text { Teste de Levantar } \\
\text { da Cadeira } \\
\text { (№ de Repetições) }\end{array}$ & $3(9,09 \%)$ & $20(60,60 \%)$ & $10(30,31 \%)$ \\
\hline $\begin{array}{c}\text { Teste de Flexão de Braço } \\
\text { (№ de Repetiçōes) }\end{array}$ & $1(3,03 \%)$ & $21(63,64 \%)$ & $11(33,33 \%)$ \\
\hline $\begin{array}{c}\text { Teste de Marcha } \\
\text { Estacionária de 2 Minutos } \\
\text { (№ de Passos) }\end{array}$ & $15(45,45 \%)$ & $18(54,55 \%)$ & - \\
\hline $\begin{array}{c}\text { Teste de Sentar } \\
\text { e Alcancar os Pés } \\
\text { (cm +l-) }\end{array}$ & $16(48,48 \%)$ & $16(48,48 \%)$ & $1(3,04 \%)$ \\
\hline $\begin{array}{c}\text { Teste de Alcançar } \\
\text { as Costas (cm+l-) }\end{array}$ & $15(45,45 \%)$ & $17(51,51 \%)$ & $1(3,04 \%)$ \\
\hline $\begin{array}{c}\text { Teste de Levantar } \\
\text { e Caminhar (segundos) }\end{array}$ & $15(45,45 \%)$ & $18(54,55 \%)$ & - \\
\hline
\end{tabular}

NOTA: n=número de sujeitos; \%=Porcentagem 
Em relação à flexibilidade de membros inferiores e superiores, o estudo evidencia certo equilíbrio entre o número de idosos na faixa dos valores aceitáveis e aqueles abaixo do considerado normal.

Um Programa de atividades físicas voltado para a terceira idade não pode prescindir de alongamentos. Estes exercícios podem servir como complemento do aquecimento ou mesmo potencializar a recuperação no final do treino. Melhoram a elasticidade muscular a mobilidade articular assim como diminuem o risco de lesões (op.cit.).

Os valores relacionados à capacidade aeróbica apresentam um quadro positivo para praticamente a metade dos avaliados, contudo, uma parcela significativa (45, 45\%) não obteve índice satisfatório.

Esses números, anteriormente, apresentados são corroborados na Tabela 3, onde verifica-se médias de escores satisfatórias no tocante à força de membros superiores e inferiores, contudo, observa-se que capacidades como flexibilidade e resistência aeróbica tiveram um desempenho inferior em função da quantidade considerável de sujeitos na faixa "abaixo da média".

TABELA 3 - Média e Desvio-Padrão dos Escores obtidos nos Testes de Aptidão Física

\begin{tabular}{|c|c|}
\hline ITEM DO TESTE & $\begin{array}{c}\text { MÉDIA E DESVIO-PADRÃO DE APTIDÃO PARA OS } \\
\text { IDOSOS PESQUISADOS N( }( \pm D P)\end{array}$ \\
\hline $\begin{array}{l}\text { Teste de Levantar da Cadeira } \\
\text { (№ de Repetições) }\end{array}$ & $15,66(5,11)$ \\
\hline $\begin{array}{l}\text { Teste de Flexão de Braço } \\
\text { (№ de Repetiçōes) }\end{array}$ & $17,75(4,70)$ \\
\hline $\begin{array}{l}\text { Teste de Marcha Estacionária } \\
\text { de } 2 \text { Minutos (№ de Passos) }\end{array}$ & $72,57(16,69)$ \\
\hline $\begin{array}{l}\text { Teste de Sentar e Alcancar } \\
\text { os Pés }(\mathrm{cm}+/-)\end{array}$ & $-5,57(9,34)$ \\
\hline $\begin{array}{l}\text { Teste de Alcançar } \\
\text { as Costas }(\mathrm{cm}+/-)\end{array}$ & $-8,36(7,94)$ \\
\hline $\begin{array}{l}\text { Teste de Levantar } \\
\text { e Caminhar (segundos) }\end{array}$ & $6,58(0,84)$ \\
\hline
\end{tabular}

NOTA: $n=m e ́ d i a, d p=$ desvio padrão

Por fim, em relação ao IMC, observou-se que a média dos participantes da pesquisa encontra-se na faixa do sobrepeso (valor maior ou igual a 27). Portanto, na faixa de risco para incapacidade ou desenvolvimento de doenças crônico-degenerativas.

\section{CONCLUSÃo}

A prática regular de exercícios físicos para a maioria dos idosos pesquisados influenciou positivamente no tocante à força de membros superiores e inferiores.

Embora uma considerável parcela da amostra tenha obtido valores satisfatórios nos demais itens analisados, observou-se, também, a necessidade de se se reavaliar as atividades relacionadas à flexibilidade, resistência aeróbica, equilíbrio e agilidade, aspectos estes com uma grande quantidade de idosos ainda na faixa "abaixo da média" para as capacidades anteriormente analisadas. 


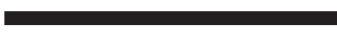

AUTORES:

Patrícia C. A. Montenegro

Eduardo L. L. Montenegro

Cláudia M. N. Santos

Gabriel Pinto Ferreira

Grupo de Estudos e Pesquisa em Docência e Formação Profissional em Educação Física, Universidade Federa de Alagoas, Brasi

\section{O estágio na formação}

1. AMORIM, F. S.; DANTAS, E. H. M. Autonomia e resistência aeróbica em idosos: efeitos do treinamento autonomia de idosos. Fitness \& Perfomance Journal, Rio de Janeiro, v. 1, n. 3, p. 47-59, 2002

2. CAMPOS, M. A. Musculação: Diabéticos, osteoporóticos, Idosos, crianças, obesos. Rio de Janeiro: Sprint, 2008.

3. DANTAS, E.H.M; VALE R.G.S Protocolo GLAM de avaliação de autonomia funcional. Fitness \& Performance Jornal, v.3, p. 175-182, 2004

4. NAHAS, M.V. Atividade Física, Saúde e Qualidade de Vida. Londrina: Midiograf, 2001

5. RIKLI, Roberta E; JONES C. Jessie. Teste de Aptidão Física para Idosos. Barueri, SP: Manole, 2008.

6. NIEMAN, David C. Exercício e Saúde: Como se prevenir de doenças usando o exercício como seu medicamento. São Paulo, SP: Manole, 1999.

\section{de professores: Visão discente}

\section{PALAVRAS CHAVE:}

Estágio supervisionado. Formação

de professores. Educação Física. Licenciatura.

\section{RESUMO}

Este estudo tem como objetivo analisar a importância do estágio supervisionado na formação inicial de professores de Educação Física na visão dos discentes do curso de educação física licenciatura da Universidade Federal de Alagoas. Este estudo é de natureza qualitativa com características descritiva. 0 grupo amostral foi composto por 17 alunos

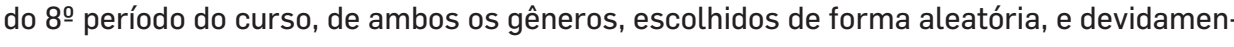
te matriculados na disciplina de estágio supervisionado IV. Como instrumento de coleta de dados foi aplicado um questionário semiestruturado. A análise dos dados foi realizada através da análise interpretativa, técnica onde se utiliza a categorização para melhor interpretar as informações dos sujeitos ${ }^{(1)}$. Os resultados desta pesquisa nos permitem concluir que os discentes do curso de educação física apontam percepções com índices regulares de concordância com relação à importância do Estágio Supervisionado na formação inicial do professor de Educação Física e também no que diz respeito às demais questões relacionadas ao Estágio Supervisionado. Os alunos acreditam que o estágio cria novas possibilidades aos discentes, e proporciona a criação de um olhar de educador no que diz respeito às questões do exercício da sua profissão relacionadas à docência. 